\title{
TOp TEn resistant Microorganisms at intensive care unit: a 2018 global expert survey (TOTEM study protocol)
}

\author{
Jordi Rello ${ }^{1,2 *}$, Leonel Lagunes ${ }^{2,3 *}$, Joana Alves ${ }^{4}$, Celine Pulcini ${ }^{5,6}$, Andrew Conway-Morris ${ }^{7}$, Emine Alp $^{8}$, \\ Jeroen Schouten ${ }^{9}$, Mervyn Mer ${ }^{10}$, Zhongheng Zhang ${ }^{11}$ \\ ${ }^{1}$ CIBER de Enfermedades Respiratorias (CIBERES), Barcelona, Spain; ${ }^{2}$ Clinical Research/Innovation in Pneumonia \& Sepsis (CRIPS) Group, Vall \\ d'Hebron Institut of Research (VHIR), Barcelona, Spain; ${ }^{3}$ Intensive Care Department, Hospital de Especialidades Médicas, San Luis Potosi, Mexico; \\ ${ }^{4}$ Infectious Diseases Department, São João Hospital Center, Porto, Portugal; ${ }^{5}$ EA 4360 APEMAC, Université de Lorraine and Infectious Diseases \\ Department, CHRU de Nancy, Nancy, France; ${ }^{6}$ European Study Group on antibiotic Policies (ESGAP), ESCMID, Basel, Switzerland; ${ }^{7}$ Wellcome \\ Trust Clinical Research Career Development Fellow, University of Cambridge, Cambridge, UK; ${ }^{8}$ Infection Control Committee, Kayseri University \\ Hospital, Kayseri, Turkey; ${ }^{9}$ Department of Intensive Care Medicine, Radboud University Medical Center, Nijmegen, The Netherlands; ${ }^{10}$ Divisions \\ of Critical Care and Pulmonology, Department of Medicine, Charlotte Maxeke Johannesburg Academic Hospital and Faculty of Health Sciences, \\ University of the Witwatersrand, Johannesburg, South Africa; ${ }^{11}$ Department of Emergency Medicine, Sir Run-Run Shaw Hospital, Zhejiang \\ University School of Medicine, Hangzhou 310000, China \\ Contributions: (I) Conception and design: J Rello, L Lagunes; (II) Administrative support: J Rello; (III) Provision of study materials or patients: All \\ authors; (IV) Collection and assembly of data: L Lagunes; (V) Data analysis and interpretation: L Lagunes, J Alves; (VI) Manuscript writing: All \\ authors; (VII) Final approval of manuscript: All authors. \\ *These authors contributed equally to this work. \\ Correspondence to: Joana Alves. Alameda Prof. Hernâni Monteiro, 4200-319 Porto, Portugal. Email: joanamargaridaalves@gmail.com.
}

Background: This global survey will provide global expert ranking of the most urgent multidrug bacteria present at the intensive care units (ICU) that have become a threat in daily clinical practice. We believe efforts on education, investigation, funding and development of new antimicrobials or new antimicrobial approach should be directed in near future. The 2018 study protocol is reported here in.

Methods: A global survey will be performed using an electronic platform (SurveyMonkey ${ }^{\circledR}$ ). The survey will compile data on key aspects of the actual threat of antimicrobial-resistant bacteria globally in the ICU.

Keywords: Multidrug-resistant (MDR) bacteria; infection; colonization; prevention; research; antimicrobials; intensive care unit (ICU); severe acute respiratory failure; sepsis and septic shock

Received: 22 December 2017; Accepted: 03 January 2018; Published: 22 January 2018.

doi: $10.21037 /$ jeccm.2018.01.03

View this article at: http://dx.doi.org/10.21037/jeccm.2018.01.03

\section{Introduction}

Multidrug-resistant (MDR) and extreme drug-resistant (XDR) bacteria have become a health priority in many national health systems, with a high burden of disease and uprising costs for attention (1,2). Many efforts have been made to prevent colonization, infection and decrease mortality, mostly in critically ill patients (3-7). However, differences regarding geographical zones, accessibility to new treatments, and differences between public and private health systems have been overlooked, along with the controversies between local and societies guidelines; some including poor quality of the evidence base, variety on aims and differences in methodology, MDR definitions and prevention measures $(8,9)$. Nevertheless, guidelines implementation is challenging especially where resources are limited. For these reasons, it is urgent to know based on a clinical base experience, which are the ten most important MDR bacteria, based on clinical presentation, severity-ofillness, impact on outcomes and resources utilization.

Recently a global priority list (PPL) of MDR bacteria was proposed to guide research, discovery and development 
of new antibiotics by the World Health Organization (WHO) (10), however critically ill patients who represents the group where these pathogens becomes an urgent threat, because of the imperativeness in taking decisions, have been mixed with other types of patients. To know which are the more representative's pathogens involved in MDR infections in critically ill patients and the possibility to ranked them is crucial to guide production of guidelines and future clinical research. With this background and due to imbalances in representation in previous priority lists, we developed the TOp TEn resistant Microorganisms (TOTEM) in critical care study group to assess this urgent matter.

The main objective of the TOTEM study is to describe assessed on expert opinion and current evidence a global list of the top ten most clinically relevant MDR/XDR bacteria involved in critically ill patients around the world. Secondary objectives are to prioritize them into three different groups: critical, high, medium according to its urgency of prevention, detection and development of new treatment strategies in a global basis, identify whether geographical location significantly influence presence of one or another MDR bacteria. This would be helpful in addressing unmet clinical needs and a research agenda, with special emphasis on respiratory infections and bacteremia causing severe acute respiratory failure, sepsis and septic shock.

\section{Methods}

Based on recent recommendations $(11,12)$ TOTEM survey was designed. A coordination steering committee of experts all involved in identification, prevention and treatment of MDR bacteria in critically ill patients will be selected, based on publications, background, geographical region and recommendations, and constitutes the TOTEM study group. An electronic survey will be created in concordance with the steering committee. The list of organisms will be based in next criteria for prioritization: all-cause mortality, healthcare and community burden, prevalence of resistance, 5-year trend of resistance, transmissibility and preventability, severity-of-illness, treatability and current pipeline.

The survey will be performed using an electronic platform (SurveyMonkey ${ }^{\circledR}$ ). This survey will be distributed by invitation from the members of the Steering Committee; it will be an online questionnaire requiring no specific data of patients, no intervention and no informed consent is required. Due to the observational aim of the study, qualifying as quality control assessment, research ethics board consultation was exempted.

In order to develop a more realistic understanding of clinical practice, we encouraged all clinicians that care for critically ill patients, with interest and experience on critical infectious diseases to response to the survey. Paediatric and neonatal intensive care units (ICUs) are excluded. It is requested that only one professional per unit complete the questionnaire, to have consistency and to avoid data multiplication. Data analyses and reports will be done with anonymization of respondents. An estimated of at least $60 \%$ of answers is expected. The main deliverable will be a PPL of resistant pathogens representing a threat for ICU adults. Regional secondary analyses will be performed.

\section{Questionnaire}

The survey will compile data on key aspects of the actual threat of antimicrobial-resistant bacteria globally in the ICU. Details of the survey are summarized in Appendix 1.

\section{Definitions}

Definition of "Resistant" organisms will be based on breakpoints provided by EUCAST recommendations (13). MDR: bacteria non-susceptibility to at least one agent in three or more antimicrobial categories: resistant to ceftazidime, cefepime, aztreonam, ciprofloxacin, piperacillin, and gentamicin. XDR: non-susceptibility to at least one agent in all but two or fewer antimicrobial categories (14). Cohorting measures: patients requiring transmission basic precautions (patients with the same single infectious agent) are placed within an area of a hospital ward with a dedicated team of healthcare staff. Formulary restriction is defined as the need of an infection control team authorization for specific antibiotic prescription (broad spectrum antibiotics). Cycling antibiotics: scheduled substitution of a class of antibiotics (or a specific member of a class) with a different class (or a specific member of that class) that exhibits a comparable spectrum of activity.

\section{Statistical analysis}

All responses to survey questions are categorical variables and will be analyzed by using descriptive statistics, reporting proportions (percentages). Chi-square test (or Fisher's exact test when appropriate) will be performed to evaluate a potential association between the geographical location 
of the participants and responses to survey questions (15). A two-tailed $\mathrm{P}$ value less than 0.05 will be considered statistically significant. In order to evaluate the presence of difference in practices according to geographical location and health care system; multivariable analysis to test the independent effect of variables on the outcome of interest will be performed. Specific data according to techniques used to determine resistance is out of the scope of this study.

\section{Aim}

To know from first-hand the most important resistant pathogens in participating ICUs. To analyze globally and regionally most clinically important resistant pathogens according to answers given by attending physicians. To rank from critical to medium urgency the list of MDR bacteria in participating ICUs. To assess knowledge on preventability measures taken into account. Finally, some recommendations where future efforts should be directed in prevention, adequate treatment and clinical trials regarding MDR infections in critically ill patients can be proposed.

\section{Acknowledgements}

None.

\section{Footnote}

Conflicts of Interest: The authors have no conflicts of interest to declare.

\section{References}

1. Tabah A, Koulenti D, Laupland K, et al. Characteristics and determinants of outcome of hospital-acquired bloodstream infections in intensive care units: the EUROBACT International Cohort Study. Intensive Care Med 2012;38:1930-45.

2. Vasudevan A, Memon BI, Mukhopadhyay A, et al. The costs of nosocomial resistant gram negative intensive care unit infections among patients with the systemic inflammatory response syndrome- a propensity matched case control study. Antimicrob Resist Infect Control 2015;4:3.

3. Zaky A, Zeliadt SB, Treggiari MM, et al. Patient-level interventions to prevent the acquisition of resistant gram- negative bacteria in critically ill patients: a systematic review. Anaesth Intensive Care 2015;43:23-33.

4. Teerawattanapong N, Kengkla K, Dilokthornsakul P, et al. Prevention and Control of Multidrug-Resistant Gram-Negative Bacteria in Adult Intensive Care Units: A Systematic Review and Network Meta-analysis. Clin Infect Dis 2017;64:S51-60.

5. Harris AD, Pineles L, Belton B, et al. Universal glove and gown use and acquisition of antibiotic-resistant bacteria in the ICU: a randomized trial. JAMA 2013;310:1571-80.

6. Huskins WC, Huckabee CM, O'Grady NP, et al. Intervention to reduce transmission of resistant bacteria in intensive care. N Engl J Med 2011;364:1407-18.

7. Derde LPG, Cooper BS, Goossens H, et al. Interventions to reduce colonisation and transmission of antimicrobialresistant bacteria in intensive care units: an interrupted time series study and cluster randomised trial. Lancet Infect Dis 2014;14:31-9.

8. Otter JA, Mutters NT, Tacconelli E, et al. Controversies in guidelines for the control of multidrug-resistant Gramnegative bacteria in EU countries. Clin Microbiol Infect 2015;21:1057-66.

9. Drees M, Pineles L, Harris AD, et al. Variation in definitions and isolation procedures for multidrug-resistant Gram-negative bacteria: a survey of the Society for Healthcare Epidemiology of America Research Network. Infect Control Hosp Epidemiol 2014;35:362-6.

10. Tacconelli E, Magrini N. Global priority list of antibioticresistant bacteria to guide research, discovery, and development of new antibiotics. Available online: http:// www.who.int/medicines/publications/WHO-PPL-Short_ Summary_25Feb-ET_NM_WHO.pdf?ua=1

11. Bennett C, Khangura S, Brehaut JC, et al. Reporting guidelines for survey research: an analysis of published guidance and reporting practices. PLoS Med 2010;8:e1001069.

12. Pulcini C, Leibovici L; CMI Editorial Office. CMI guidance for authors of surveys. Clin Microbiol Infect 2016;22:901-2.

13. European Committee on Antimicrobial Susceptibility Testing: Breakpoint tables for interpretation of MICs and zone diameters Version 7.1, 2017. Available online: http:// www.eucast.org/fileadmin/src/media/PDFs/EUCAST_ files/Breakpoint_tables/v_7.1_Breakpoint_Tables.pdf

14. Magiorakos AP, Srinivasan A, Carey RB, et al. Multidrugresistant, extensively drug-resistant and pandrug-resistant 
bacteria: an international expert proposal for interim standard definitions for acquired resistance. Clin Microbiol Infect 2012;18:268-81.

doi: 10.21037/jeccm.2018.01.03

Cite this article as: Rello J, Lagunes L, Alves J, Pulcini C, Conway-Morris A, Alp E, Schouten J, Mer M, Zhang Z. TOp TEn resistant Microorganisms at intensive care unit: a 2018 global expert survey (TOTEM Study protocol). J Emerg Crit Care Med 2018;2:6.
15. Zhang Z. Univariate description and bivariate statistical inference: the first step delving into data. Ann Transl Med 2016;4:91. 


\section{Contact details}

1. Name/initials of the person answering the questionnaire*:

2. E-mail:

3. Name of the unit:

4. Name of the hospital:

5. Private/public:

6. Academic-training/non-academic:

7. City/rural:

8. Country/province:

\section{Professional information}

9. Do you belong to any of these societies?
A) ESCMID.
B) ESICM.
C) ATS/IDSA.
D) National Society of Critical Care (name).
E) Panamerican Society of Infectious Diseases.

10. Primary specialty:
A) Internal medicine.
B) Anaesthesiology.
C) Emergency medicine.
D) Surgery.
E) Infectious disease.
F) Clinical pharmacology.
G) Microbiology.
H) Preventive medicine and epidemiology/public health.
I) Intensive care medicine.

11. Name your ICU sub-specialty:
A) Trauma.
B) Cardiac surgery.
C) Medical-surgical.
D) Other.

13. Does your unit admit $>5 \%$ :
A) Transplant patients ( ).
B) Haematological patients ( ).
C) Burn patients ( ).
D) No immunocompromised patients ( ).

13. Number of beds in your ICU:

14. Nurse/HCP ratio: in your ICU: _ _

15. Experience in ICU infections:
A) 1-5 years.
B) 5-10 years.
C) $>10$ years.

\section{TOTEM indicators}

16. In the last 5 years have you noticed an increase in community-acquired infections due to MDR bacteria admitted to your ICU?
A) Yes.
B) No.

17. In the last 5 years have you noticed an increase in MDR bacteria on hospital-acquired infections admitted to your ICU?
A) Yes.
B) No.

18. In your experience are resistant infections associated with higher mortality non-resistant ones?
A) Yes.
B) No.

19. If your answer was Yes; is it because:

A) Resistant pathogens are more virulent.

B) Correct initial treatment is more difficult.

C) More severely ill patients develop resistant infections.

D) None of the above.

E) All of the above.

20. When a resistant pathogen is detected in your ICU; are prevention measures implemented (as indicated in your local/national guidelines)?
A) Yes, almost always.
B) Yes, sometimes.
C) No.

21. Specify witch pathogen is the main concern in your unit (mark only one):

A) Carbapenemase expressing Klebsiella pneumoniae (KPC) ( ).

B) Carbapenem-resistant Acinetobacter baumannii ( ).

C) Carbapenem-resistant Pseudomonas aeruginosa ( ).

D) Oxacillin/methicillin-resistant Staphylococcus aureus (MRSA) ( ).

E) Extended-spectrum beta-lactamase producing Enterobacteriaceae (ESBL).

22. According to your previously answer, which of the following are taken into (you can mark more than one).

A) Contact restriction measures ( ).

B) Air restriction measures/negative pressure rooms ( ).

C) Enhanced hand wash measures ( ).

D) Cultures from possible carriage sites ( ).

E) Notify Epidemiologist and/or Nosocomial

*These details are only to identify duplicates. Data collection, analysis and report will be anonymised. 
infections committee ( ).

F) Educational measures to staff, nurses, fellows/ junior doctors, students, etc. ( ).

G) Isolation / cohort measures ( ).

23. In your experience, please rank from 1 to 5 (1: most urgent; 5: less urgent) the measures that could limit the development and spread of antibiotic resistance:

* Cohort

* Increase nurse/HCP ratio

* Infectious diseases/epidemiology/other interconsultation

* Formulary restriction _

* De-escalation

24. In your experience, which measures do you believe are most effective in preventing MDR development and spread (rank 1 to 5 )

* Antibiotic de-escalation:

* Cycling of antibiotics:

* Education programs:

* Formulary restriction:

* Infectious disease consultation:

25. Please rank from 1 to 5 (1: most concern; 5: less concern) regarding your level of concern for emerging resistance to the following groups of antibiotics:

* Quinolones:

* 3rd or 4th generation Cephalosporins:

* Carbapenems:

* Beta lactam inhibitors (pip/taz):

* Glycopeptides:

26. Please order from 1 to 10 (1: most common; 10: less common) according to prevalence of isolated pathogens) in your ICU in 2017 (patients with a confirmed infection):

* Oxacillin/methicillin-resistant Staphylococcus aureus (MRSA)

- Vancomycin-resistant Enterococci (VRE)

* Extended spectrum beta-lactamases (ESBL) Klebsiella pneumoniae

* Extended spectrum beta-lactamases (ESBL) Escherichia coli

* XDR Pseudomonas aeruginosa

* Carbapenem-resistant Pseudomonas aeruginosa

* Carbapenem-resistant Acinetobacter baumannii _

* Carbapenem-resistant Klebsiella pneumoniae

* Trimethoprim-sulfamethoxazole/septrin (TMPSMX)-resistant Stenotrophomonas maltophilia

- AmpC hyperproductor Enterobacteriaceae

27. Locally, in your unit, are there any other resistant microorganism you believe due to frequency, mortality or difficulties in adequate initial treatment should be included in list?

A) Yes.

B) No.

28. If previous answer was affirmative, please identify it:

29. Please order from 1 to 10 (1: most lethal; 10: less lethal) according to related mortality from your perspective in your ICU in the last year:

* Oxacillin/methicillin-resistant Staphylococcus aureus (MRSA)

* Vancomycin-resistant Enterococci (VRE)

* Extended spectrum beta-lactamases (ESBL) Klebsiella pneumoniae

* Extended spectrum beta-lactamases (ESBL) Escherichia coli

* Multidrug-resistant Pseudomonas aeruginosa

* Carbapenem-resistant Pseudomonas aeruginosa

* Carbapenem-resistant Acinetobacter baumannii _

* Carbapenem-resistant Klebsiella pneumoniae

- TMP-SMX-resistant Stenotrophomonas maltophilia

* Extended spectrum beta-lactamases (ESBL) Serratia spp _

30. Please order from 1 to 10 (1: most difficult, 10: less difficult) adequate treatment availability; from your perspective in your ICU in the last year:

* Oxacillin/methicillin-resistant Staphylococcus aureus (MRSA) _

* Vancomycin-resistant Enterococci (VRE) _

* Extended spectrum beta-lactamases (ESBL) Klebsiella pneumoniae

* Extended spectrum beta-lactamases (ESBL) Escherichia coli

* Multidrug-resistant Pseudomonas aeruginosa _

* Carbapenem-resistant Pseudomonas aeruginosa

* Carbapenem-resistant Acinetobacter baumannii _

* Carbapenem-resistant Klebsiella pneumoniae

* TMP-SMX-resistant Stenotrophomonas maltophilia _

* Extended spectrum beta-lactamases (ESBL) Serratia spp

31. Regarding community-acquired pneumonia please rank from 1 to 5 most common resistant pathogens isolated in your ICU:

* 3rd generation cephalosporins-resistant Streptococcus pneumoniae

* Macrolide-resistant Streptococcus pneumoniae _

* 3rd generation cephalosporins-resistant 
Enterobacteriaceae

* Quinolones-resistant Pseudomonas aeruginosa _

* Oxacillin/methicillin-resistant Staphylococcus aureus (MRSA)

32. Regarding hospital-acquired pneumonia (and VAP) please rank from 1 to 5 most common resistant pathogens isolated in your ICU:

* Multidrug-resistant Pseudomonas aeruginosa _

* Carbapenem-resistant Pseudomonas aeruginosa

* Carbapenem-resistant Acinetobacter baumannii _

* Carbapenem-resistant Klebsiella pneumoniae _

* Oxacillin/methicillin-resistant Stapbylococcus aureus (MRSA)

33. Regarding intraabdominal infections please rank from 1 to 5 most common resistant pathogens isolated in your ICU:

* Vancomycin-resistant Enterococci _

* Multidrug-resistant Pseudomonas aeruginosa _

* Multidrug-resistant Escherichia coli

* Carbapenem-resistant Acinetobacter baumannii
* Carbapenem-resistant Klebsiella pneumoniae

34. Regarding nosocomial soft tissue and skin infections please rank from 1 to 5 most common resistant pathogens isolated in your ICU:

- Oxacillin/methicillin-resistant Staphylococcus aureus (MRSA)

* Vancomycin-resistant Enterococci

* Multidrug-resistant Pseudomonas aeruginosa _

* Multidrug-resistant Escherichia coli

* Carbapenem-resistant Klebsiella pneumoniae

35. Regarding nosocomial catheter-associated bacteremia please rank from 1 to 5 most common resistant pathogens isolated in your ICU:

* Oxacillin/methicillin-resistant Staphylococcus aureus (MRSA)

* Vancomycin-resistant Enterococci _

* Multidrug-resistant Pseudomonas aeruginosa _

* Multidrug-resistant Escherichia coli

* Carbapenem-resistant Klebsiella pneumoniae 